\title{
Dynamic in vitro hemocompatibility testing - improving the signal to noise ratio
}

\author{
M. Mueller ${ }^{1}$, B. Krolitzki ${ }^{2}$, B. Glasmacher ${ }^{2}$ \\ ${ }^{1}$ Institute for Multiphase Processes, Leibniz University Hannover, mueller@imp.uni-hannover.de \\ ${ }^{2}$ Institute for Multiphase Processes, Leibniz University Hannover
}

\begin{abstract}
Assigning the hemocompatibility of small vascular implants is one of the great challenges in biomedical engineering. Due to the fact, that there are no widely approved test setups ${ }^{1}$, we decided to developed a modified CHANDLER-Loop system for dynamic in vitro hemocompatibility tests. The setup allows simultaneous testing of about 30 tube rings with an inner diameter of 2,4 mm. Cardiovascular implants can be placed into these tube rings. After filling them with anticoagulated blood and closing them to loops, they were placed on a rotating disc. While the loop is rotating, the blood remains in the lower part of the loop. The relative movement between the foreign surface and the blood sample induces a blood flow without the need of mechanical pumping. A first test series with the new test setup had been conducted to achieve a highly hemocompatible tube material. The results were compared to hemocompatibility tests done by LEMM, mentioned in the ISO 10993-4,3. We could prove, that Tygon S5OHL ${ }^{\circledR}$ and Polyurethane achieve similar hemocompatibility values. In addition, we did in vitro tests of bare metal stents. We recorded a difference in the index of thrombocytes of about $17 \%$ between the loops with three stents and the control. During further experiments the difference decreases to $8 \%$, which might be due to adhesion processes. The ability to measure these processes demonstrates the high sensitivity and very low background activation of the test setup. This can be assured by the recorded index of hemolysis, which had been less than $0,8 \%$ in all our experiments.

By developing and improving our modified CHANDLER-Loop system, we were able to establish a dynamic in vitro test setup for the hemocompatibility testing of small vascular implants. Because of its extremely low background activation and a high variability, the system serves as a benchmark for upcoming test setups.
\end{abstract}

\section{Introduction}

Approximately 4 million stents are implanted annually worldwide $^{4}$. The resultant foreign surface contact leads to an activation of the human coagulation system, which is normally inhibited by the application of anticoagulants. Due to these interactions between implants and human blood flow, materials used for the fabrication of cardiovascular devices must be highly hemocompatible. According to the ISO 10993-4 dynamic in vitro test setups are suitable screening methods to assign the hemocompatibility of small vascular implants ${ }^{3}$. A great challenge in the development of such test setups lies in the ratio between the small test material surfaces and the surfaces of components used for the blood handling. A background activation caused by these components may overlay the implant signal, so that a measurable implant effect is reduced ${ }^{5}$. In addition, there are no widely approved test setups or an agreed list of hemocompatible materials ${ }^{1}$. Moreover the reproducibility of dynamic hemocompatibility tests is made difficult due to missing information about test parameters and conditions ${ }^{6}$.

Because of its low background activation, which is realized by inducing a blood flow without the need of a mechanical pump system, the CHANDLER-Loop is a widely used setup for hemocompatibility testing of small vascular implants ${ }^{7}$. For this reason we decided to develop a modified CHANDLER-Loop, which should be used as a benchmark system for more complex in vitro setups.

\subsection{In vitro hemocompatibility testing}

The blood contact to a foreign surface is followed by a rapid plasma protein adsorption, which leads to the activation of the coagulation system. In addition, the surface contact or mechanical forces can cause a damage of the red blood cell membrane, which results in hemolysis. As previously mentioned, these surface interactions occur at the implant materials as well as at the blood handling components. Because of this, the blood damage, which is induced by the test setup, should be very low and well defined.

In 1958 CHANDLER developed a test system for analyzing the coagulation system. Native whole blood was filled into tubes, which were closed to loops. While rotating on a disc which is held in a $23^{\circ}$ angle, the blood remains in the lower part of the loop, which results in a relative movement between tube surface and blood. By measuring the time until the tube was clotted through a thrombus, CHANDLER was able to analyze the functionality of the coagulation system.

\subsection{Modified ChANDLER-Loop}

The developed tube ring holder can be connected to a common roller pump (BVP Standard, ISMATEC), which facilitates an accurate adjustment of the rotation speed and a hemocompatibility testing in almost every laboratory. The whole setup is placed in a chamber, which is regulated at $37^{\circ} \mathrm{C}$. 
A disc with a diameter of $220 \mathrm{~mm}$ is mounted on mandrel, which is part of the constructed connection plate between test setup and roller pump.
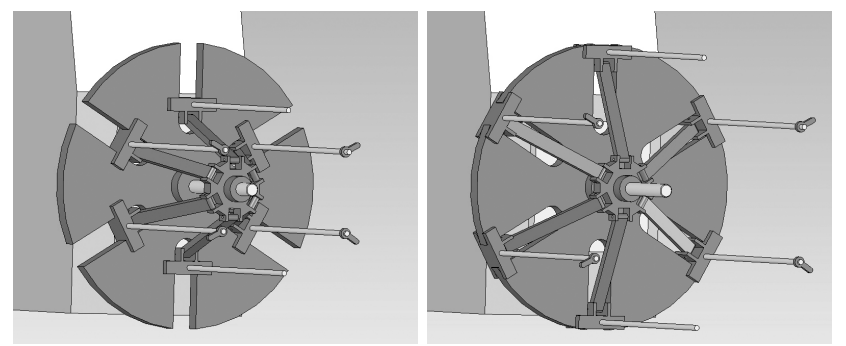

Figure 1 Adjustable tube ring holder, shown for the minimum diameter (left) and the maximum diameter (right).

The developed mechanism allows a variable radial movement of stainless steel pins with a length of $150 \mathrm{~mm}$. This realizes a simultaneous rotation of up to 30 specimen with a various loop diameter between $110-220 \mathrm{~mm}$, which approves a comparison between different materials or in case of implant testing, the reference to a control. Moreover, by removing loops from the test setup, it is possible to analyze blood samples at different points of the testing time.

\section{Materials and Methods}

Regarding the direct blood contact of the tube rings used as blood handling system, we had to investigate a highly hemocompatible tube material to minimize the background activation, which otherwise may overlay our implant signal. Our first experiment was designed in reference to a hemocompatibility test series, which was conducted by $\mathrm{LEMM}^{2,8}$. While LEMM performed his tests with tubes made of Polyethylene, Polyurethane, Silicone, Polyvinylchloride (plasticized / unplasticized) and Polypropylene, we chose tubes the following materials:

- $\quad$ Silicone (SIK 8363, RAUMEDIC)

- Polyvinylchloride (PVC Guttasyn TOL)

- Tygon B44-EU $U^{\circledR}$ noDOP

- Tygon S5OHL ${ }^{\circledR}$ medical tubing (both SAINTGOBAIN - PERFORMANCE Plastics)

LEMMS setup was quite similar to a CHANDLER-Loop system and by taking the same surface/volume ratio $(251 / \mathrm{cm})$ and flow velocity $(18,8 \mathrm{~cm} / \mathrm{s})$, we were able to facilitate a good comparability. While choosing the same inner diameter of $4 \mathrm{~mm}$ we had to reduce the blood volume to $3 \mathrm{ml}$ and the tube length to $61 \mathrm{~cm}$.

A second test series with bare metal stents was performed to prove the sensitivity of our new dynamic test setup, by showing that the blood damage, induced by the blood handling components, still allows a measureable implant signal. The stents were placed in tubes of Polyurethane (Ellastollan $^{\circledR}, \mathrm{BASF}$ ) with an inner diameter of $2,4 \mathrm{~mm}$ and a length of $68 \mathrm{~cm}$. We prepared three different loop types, one without any stent, serving as control for the background activation, loops containing one stent $(1=19 \mathrm{~mm})$ and loops with three stents $(2 \times 1=18 \mathrm{~mm}$ and $1 \times 1=$ $19 \mathrm{~mm})$. After every experiment the loops were washed with Triton X 100 (CARL ROTH GMBH \& CO. KG) to allow further test runs.

Tubes were filled with $3 \mathrm{ml}(\varnothing=4 \mathrm{~mm})$ or $1,5 \mathrm{ml}$ $(\varnothing=2,4 \mathrm{~mm})$ anticoagulated porcine blood (heparin, $5 \mathrm{IU} / \mathrm{ml}$ blood) and closed to a loop by using $2-4 \mathrm{~cm}$ long tubes, whose inner diameter were of the same dimension as the outer diameter of the tested tubes. After the completed test duration, blood was taken out of the tubes and the parameters hematocrit, hemolysis and thrombocyte count were determined. $60 \mu 1$ of whole blood was filled into small capillaries (BRAND GMBH \& CO. KG) and after a following centrifugation step $(11.000 \mathrm{~g}, 4 \mathrm{~min})$ the hematocrit could be noted. These values were related to the zero value, which allows a compensation of physiological variations in the blood samples and improves the comparability between different test days. Referring to the ISO 10993-4 hemolysis is a standard parameter that should be determined while testing the hemocompatibility of biomaterials ${ }^{3}$. The index of hemolysis is defined by the following formula':

$$
I H[\%]=\frac{\left(1-\frac{H C T}{100}\right) \cdot P H b}{T H b} \cdot 100 \%
$$

- $\mathrm{IH}=$ index of hemolysis $[\%]$

- $\quad \mathrm{HCT}=$ Hematocrit [-]

- $\quad \mathrm{PHb}=$ Plasma hemoglobin $[\mathrm{g} / \mathrm{dl}]$

- $\quad \mathrm{THb}=$ Total hemoglobin $[\mathrm{g} / \mathrm{dl}]$

The total hemoglobin represents the amount of hemoglobin in the whole blood, while the plasma hemoglobin describes the free hemoglobin in the blood plasma. An increasing value of plasma hemoglobin indicates a damage of the red blood cell membrane, which causes a release of hemoglobin. Both parameters were measured with a Photometer by using a test solution (Hemoglobin FS, DIASYS). To assign the activation of the coagulation system we did a thrombocyte count of every blood sample. Therefore $20 \mu 1$ of whole blood were mixed with a dyeing solution (TVTThrombozyten-Einzeltest, KABE) and after a short incubation of about $3 \mathrm{~min}$ the mixture was injected into a glass chamber (Neubauerkammer, PAUL MARIENFELD GMBH \& CO. KG), which was used for the cell counting. The counted cell numbers were related to the zero value by using the following index:

$$
T I[\%]=\frac{T C_{X}}{T C_{o}} \cdot 100 \%
$$

- $\quad \mathrm{TI}=$ Index of thrombocytes [\%]

- $\quad \mathrm{TC}_{\mathrm{X}}=$ Thrombocyte count at point $\mathrm{x}[1 / \mu 1]$

- $\quad \mathrm{TC}_{0}=$ Zero value $[1 / \mu 1]$

A decrease in the index might be caused by an adhesion of cells to the foreign surface or cell aggregation. 


\section{$3 \quad$ Results}

The values of the hematocrit showed neither an increase nor a decrease for all tested materials or implants. Furthermore, the index of hemolysis had always been less than $0,8 \%$, from which reason the results were not presented in detail.

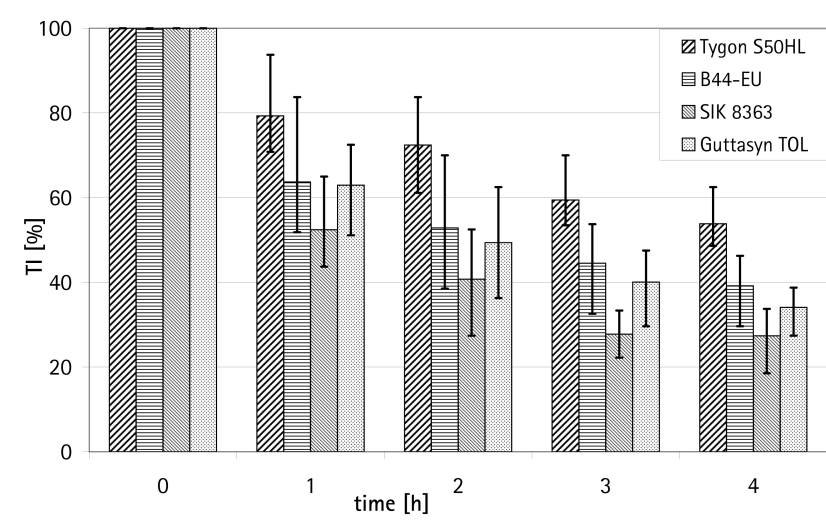

Figure 2: Index of thrombocytes, shown for all tested materials. Samples were taken at 1,2,3 and 4 hours $(n=3)$.

We could observe the greatest decrease of thrombocytes within the first hour of our experiment. The best result was recorded with a $20 \%$ lowering for Tygon S5OHL ${ }^{\circledR}$. After four hours of testing, Tygon $550 H L^{\circledR}$ showed the lowest loss of thrombocytes, while SIK 8363 induced the greatest decrease. The results of the two other materials looked similar.

Figure 3 represents the results of the bare metal stent tests with increasing number of test days. We compared the values of the control (no Stent) and the loops with three stents, because their differences were more pronounced. Within the first hour the loss of thrombocytes is about $20 \%$ for the control. Furthermore the loops with three stents are showing a decrease of another $17 \%$ compared to control. The difference is reduced with the increasing number of test days.

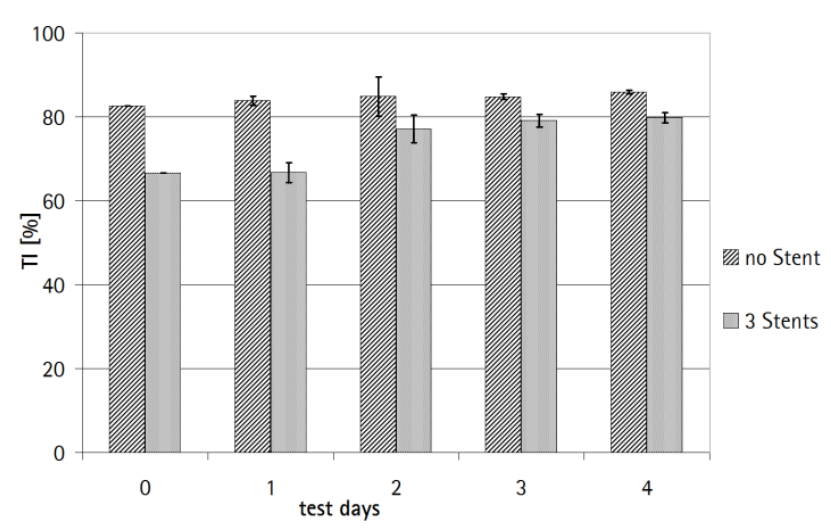

Figure 3: Index of thrombocytes, shown for the loops with three stents and the control. Samples were taken after one hour of dynamic testing $(n=5)$.

\section{Discussion}

Regarding constant values of the hematocrit measurement and the very low index of hemolysis, we could prove that our system induces an extremely low background activation. In comparison to the hemocompatibility tests performed by LEMM, we achieved similar results for SIK 8363 , which was marked with a decrease of thrombocytes of about $50 \%$ after one hour. The smallest loss of thrombocytes could be observed for Tygon $S 50 H L^{\circledR}$, whose hemocompatibility, compared to the results from LEMM, is almost equal hemocompatibility to Polyurethane. Regarding the great decrease in thrombocytes during the first hour, followed by smaller steps of decrease over the time, we could prove the statement of BLACKSHEAR \& BLACKSHEAR whereas the materials surface has the largest influence on the blood damage during the first test hour ${ }^{10}$.

Considering the results of the bare metal stent testing, we recorded meaningful differences between control and loops with three stents. This measurable effect, induced from small cardiovascular implants, demonstrates the high sensitivity of the in vitro test setup, which was already mentioned by CHRISTENSEN ET. AL, who stated that the CHANDLER-Loop is effective test model for assigning hemocompatibility of stents or vascular grafts ${ }^{11}$.

Due to further development of our modified CHANDLERLoop, we could establish a benchmark system for dynamic for hemocompatibility testing at the Institute for Multiphase Processes. Because of the very low background activation combined with its variable specimen holder, the modified CHANDLER-Loop is an ideal test setup for dynamic hemocompatibility testing of small vascular implants.

\section{Acknowledgement:}

This work is partially funded by the German Research Foundation (SFB-TR37 - Q2 and B4) and the cluster of excellence REBIRTH (EXC 62/1).

\section{$5 \quad$ References}

[1] Ratner, B.D.: Blood compatibility - a perspective. In: Journal of Biomaterials Science 11 (2000), 1107 $-1119$

[2] Lemm, Wilfried (Hrsg.): The Reference Materials of the European Communities. Kluwer Academic Publishers, 1992

[3] Selection of tests for interactions with blood. 2002 (ISO 10993-4)

[4] Ratner, B.D.: The catastrophe revisited: Blood compatibility in the 21 st Century. In: Biomaterials 28 (2007), S. 5144-5147

[5] Ratner, B.D.: The blood compatibility catastrophe. In: Journal of Biomedical Materials Research 27 (1993), S. 283-287 
[6] Seyfert, U. T. ; et. al: In vitro hemocompatibility testing of biomaterials according to the ISO 109934. In: Biomolecular Engineering 19 (2002), S. 91-96

[7] Chandler, A. B. ; Jacobsen, C. D.: In Vitro Thrombosis on Thrombotic and Hemorrhagic Diseases. In: Scandinavian Journal of Clinical \& Laboratory Investigation (1967), S. 129-139

[8] Dawids, S. (Hrsg.): Test procedures for the blood compatibility of biomaterials. Kluwer, 1993

[9] Paul, R.: Investigation of flow and material induced hemolysis with a Couette type high shear system. Helmholtz-Institut für Biomedizinische Technik an der RWTH Aachen, dissertation, 2000

[10] Skalak, R. ; Chien, S .: Handbook of Bioengineering. Mc Graw-Hill Book Company, 1987

[11] Christensen, Kjeld ; et al.: Heparin coating of stent graft - effects on platelets, coagulation and complement activation. In: Biomaterials 22 (2001), S. $349-$ 355

[12] Tschaut, Rudolf J. (Hrsg.): Extrakorporale Zirkulation in Theorie und Praxis. Pabst Science Publishers, 1999

[13] Wintermantel, E. ; Ha, S.-W.: Biokompatible Werkstoffe und Bauweisen - Implantate für Medizin und Umwelt. Springer Verlag Berlin Heidelberg, 1996 